\title{
Ionospheric Sounding Using Coded Pulse Signals ${ }^{1}$
}

\author{
D. C. Coll and J. R. Storey

\begin{abstract}
Contribution From Department of National Defence, Defence Research Board, Defence Research Telecommunications Establishment, Shirley Bay, Ottawa 4, Ontario, Can.
\end{abstract}

(Received April 8, 1964)

\begin{abstract}
At present, ionospheric sounders rely on the transmission of high peak-pulse-power to provide for the detection of received echoes. To allow for the detection of signals with poor signal-to-noise ratios, the transmitted energy must be increased. It can be assumed that significant increases are not likely to be obtained through increases in the peak transmitted power. Also, any increase in transmitted energy achieved through a simple increase in pulse duration is accompanied by a corresponding loss of range resolution. Pulse compression signals provide an increase in transmitted energy, without an increase of peak power, and without a loss of range resolution.

This paper describes an application of pulse-coded pulse compression signals to the field of ionospheric sounding. The signals used and their properties, their generation and reception, and the performance of an experimental vertical sounder are discussed. The effects of ionospheric and system perturbations on performance are also considered.
\end{abstract}

\section{Introduction}

The use of pulse sounding techniques for ionospheric investigation is now widespread. Sounding is employed for scientific purposes, frequency prediction purposes, and for real-time assistance to $\mathrm{HF}$ communication systems. Pulse transmitters are used in conjunction with suitable receivers to vertically sound both the bottom and top of the ionosphere, and to obliquely sound long-distance ionospheric paths between points on the earth's surface. By employing precise timing units for the generation of the pulse repetition rate, as well as for frequency control, the transmitter and receiver are maintained in synchronism as they are swept across a portion of the high frequency spectrum (3 to $30 \mathrm{Mc} / \mathrm{s})$.

The Defence Research Telecommunications Establishment (DRTE) has had an interest in ionospheric sounding for many years [Cox and Davies, 1955; Chapman, Davies, and Littlewood, 1955; Hatton, 1961; Jull, Doyle, Irvine, and Murray, 1962], and has been concerned with the implementation of signal processing techniques for improving the performance of sounders [Coll and Poaps, 1964].

Sounders normally rely upon the transmission of a pulse of RF energy with a short duration, to provide the desired resolution between ionospheric propagation modes; and with a high peak power, to provide the desired system sensitivity. Because of the use of narrow pulses, sounding receiver bandwidths of 30 to $100 \mathrm{kc} / \mathrm{s}$ are common; therefore, sounding receivers tend to be more susceptible to interference than normal HF communications equipment.

1 Paper presented at the 1963 Fall URSI Meeting, Seattle, Wash., Dec. 9-11,
For reception of sounding signals in high interference environments, or when the signals are low, it is necessary to increase the energy in the transmitted signal. In the past, increased transmitted energy has been achieved by increases in the peak pulse-power of the transmitter, until, now, peak powers of the order of 100 to $200 \mathrm{~kW}$ are not uncommon. It does not appear that significant increases in transmitted energy can be obtained through increased peak powers since severe difficulties arise in the design of transmitters, tunable over the $\mathrm{HF}$ band, if the peak power is over $100 \mathrm{~kW}$. Also, transmitters with such powers can cause undesirable local interference.

It is possible to increase the transmitted energy by increasing the pulse length. This has two adverse effects: first, the range resolution of the shorter pulse has been lost; second, the energy of the longer pulse is contained in a narrower bandwidth and thus will cause more interference to communication systems operating on the same frequencies.

A solution that has been implemented at DRTE is the use of coded pulse signals, i.e., signals consisting of trains of contiguous, rectangular, pulses in which each component pulse is suitably coded. Such signals can have an energy comparable to that of a long pulse, and have resolution similar to that of a single pulse with a duration equal to that of one of the component pulses of the train.

\section{Coded Pulse Signals}

The signals used in the sounding system consist of trains of component pulses. A matched filter is used in the sounding receiver to maximize the signal-towhite-noise ratio. The response of this matched filter, with the signal as an input, will determine the 
advantage of the pulse compression signals over a single pulse signal. The output of the matched filter, due to signal alone, is the autocorrelation function of the signal; and the output signal-to-noise ratio depends only on the energy in the signal and the power spectral density of the noise [Turin, 1960]. It is necessary, then, to design signals whose autocorrelation functions approximate that of a single pulse. Unlike the general radar case, there does not appear to be a requirement on sounding signals for Doppler resolution.

A signal that consists of a train of $N$ pulses may be represented by a sequence of elements, $a_{i}$, where the $a_{i}$ are the signals in each pulse. The autocorrelation function, $\rho(k)$, of the signal is given by the expression

$$
\rho(k)=\left\{\begin{array}{lr}
\sum_{i=0}^{N-1-k} a_{i} a_{i+k} & 0 \leq k \leq N-1 \\
0 & k>N-1
\end{array}\right.
$$

and

$$
\rho(-k)=\rho(k) .
$$

If the response of the system may be considered, for all practical purposes, to exist for only one component pulse duration (i.e., only for $k=0$ ), then the signal will have the same range resolution as a single pulse with a duration equal to that of a component pulse. In terms of the expression above, it is desirable that the autocorrelation function be made small for all nonzero values of $k$. The values of $\rho(k)$ for $k \neq 0$ are called the side lobes of $\rho(k)$, because of the similarity between the desired form of the autocorrelation function and a narrow-beam antenna pattern. The value of $\rho(0)$ is equal to the energy in the signal, and should be maximized relative to the side lobes. If the side lobes are low, the possibility of confusing a low-level multipath echo with a side lobe caused by a stronger echo is minimized. That is, ambiguities between side lobes in the response and multipath echoes are minimized. Two parameters that are used to compare signals are the peak-to-maximum side-lobe ratio, and the energy of the signal relative to that of a single component pulse with the same peak power.

The design of pulse signals, in which either the amplitude or the phase of the individual pulses has been coded, has received considerable attention in the literature ['Turin, 1960; Huffman, 1962; and Frank, 1963]. In particular those signals in which the $a_{i}$ are constrained to be either +1 or -1 have been thoroughly investigated. Such binary codes are particularly simple to generate. Signals in which the $a_{i}$ are allowed to be any complex number have been discussed by Huffman [1962]. He has specified necessary and sufficient conditions such that $\rho(k)=0$ for $k \neq 0$ or $(N-1)$. Huffman has called these signals "impulse-equivalent pulse trains." For convenience, they are referred to in this paper as "Huffman" signals. It is also possible to restrict the $a_{i}$ to be elements from a finite set of unit vectors, each with a distinct phase [Frank, 1963]. Such signals are called polyphase codes.

\section{Experimental System}

The specific signals used in the experimental sounding system were:

(a) Single pulses,

(b) binary codes of length 17 , and

(c) impulse-equivalent pulse trains of length 17 . The basic pulse length used was $25 \mu \mathrm{sec}$, and the signals were transmitted as double-side-band suppressed-carrier at a peak power of $1 \mathrm{~kW}$.

The single pulse and binary waveforms are shown in figure 1 . The single pulse, of amplitude +1 and duration $T$ seconds, and one-half of its (symmetrical) autocorrelation function are shown on the bottom right-hand side of the figure. The binary pulse train, of amplitude +1 or -1 and overall duration $17 T$, along with its autocorrelation function, are shown to scale. The peak value of the correlation function (equal to the energy of the signal and the peak output of the matched filter) is 17 , compared to 1 for the single pulse. The side lobes, values of the correlation function for nonzero shifts, can be seen. The peak-to-maximum side-lobe ratio is 17 to 2 or $8.5: 1$.

The impulse equivalent pulse train is shown in figure 2 , with one-half of its autocorrelation function. This signal has only one component pulse with a magnitude of 1 and hence has less energy than the constant magnitude binary signal. The energy in the Huffman signal is 7.28 times that of a single pulse of the same peak amplitude. The peak-tomaximum side-lobe ratio ${ }^{2}$ is 10.8 . The simple form of the autocorrelation function is very evident in figure 2. Note that multipath echoes would be detectable without ambiguity, as long as they did not fall upon the single side lobe.

2 The Huffman signal of length 17 with maximum energy (and peak amplitude 1) has an energy of 8.26 times that of a single pulse and a peak-to-side lobe ratio of 8.26 .

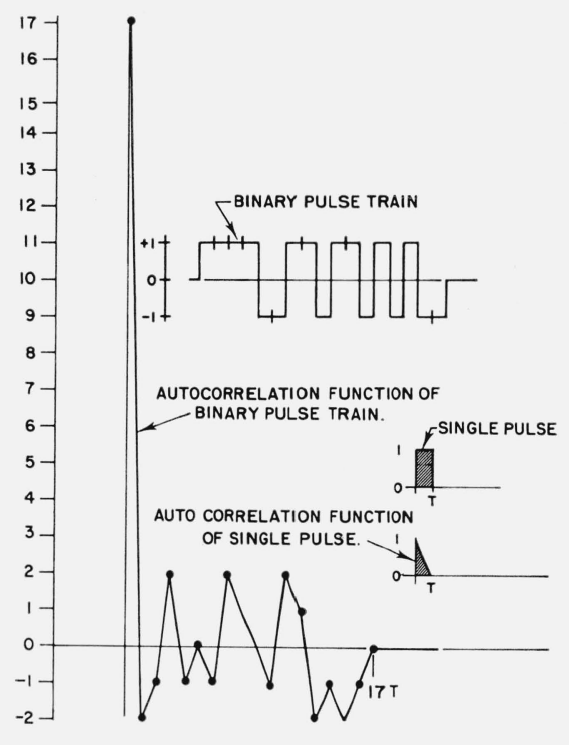

Figure 1. Single pulse and binary signals. 
One reason for the interest in pulse train signals is the simplicity with which they may be generated and received. The signals are generated in a tapped shift-register delay line, as shown in figure 3 . A single logical 1 is shifted down a 16 element shift register at a $40 \mathrm{kc} / \mathrm{s}$ shift rate. This places a voltage successively on each of 17 taps. These voltages are summed with the appropriate weighting to form the signal. The signal is then used to modulate a carrier in a balanced modulator which feeds a wideband linear power amplifier. The carrier frequency can be tuned from 2 to $24 \mathrm{Mc} / \mathrm{s}$.

Because of the bipolar nature of the signal a phase sensitive receiver must be used. Filters, matched to the low-pass signal, are used in a quadrature detector as shown in figure 4 . In this receiver, the IF signal, $f(t) \cos (w t+\theta)$ is multiplied by signals from a local oscillator in two separate phase-sensitive detectors. These local oscillator signals are in phase quadrature. [In the figure it is assumed that there is a frequency difference $\Delta \omega$, and a phase shift $\theta$, between the received signal (at the IF output) and the local oscillator:] The low-pass output of the phase-sensitive-detector in each arm is applied to identical filters, matched to the signal $f(t)$. The filter outputs are squared and added. If the frequency difference is negligible, the receiver output will be the squared correlation function.

The matched filter is shown in figure 5. It consists of a finite-time-integrator (matched to a single, $25 \mu$ sec, rectangular pulse) followed by a linear, tapped, delay line. The gain of each tap is adjusted so that the impulse response of the matched filter is the time inverse of the signal.

The waveforms generated in the experimental system are shown in figure 6 along with the corresponding matched filter outputs. In the case of the Huffman signal, it can be seen from the photograph that any side lobes caused by departures of the system from the ideal, are less than the legitimate side lobes.
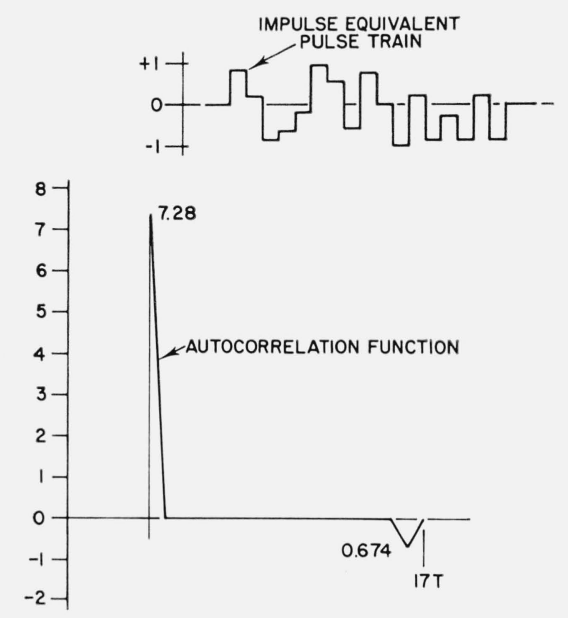

Figure 2. Impulse equivalent pulse train.

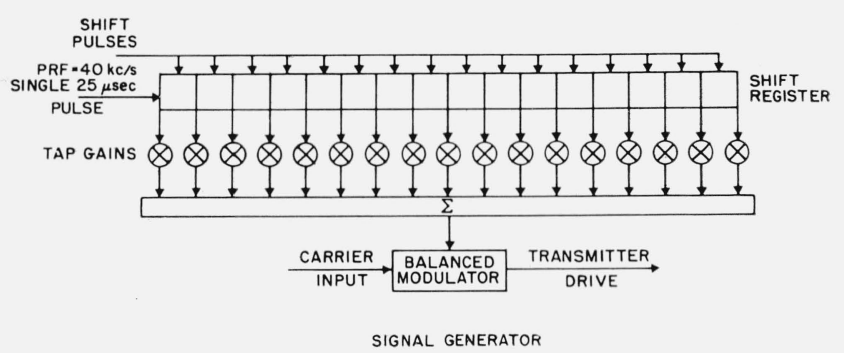

Figure 3. Signal generator.

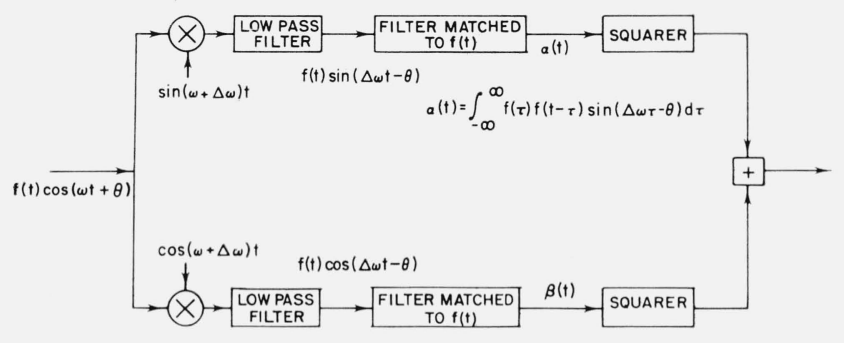

Figure 4. Receiver.

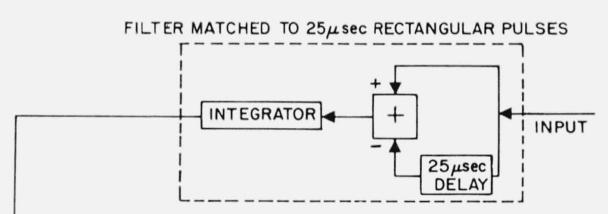

$400 \mu \mathrm{sec}$ ANALOG DELAY LINE TAPPED AT $25 \mu \mathrm{sec}$ INTERVALS

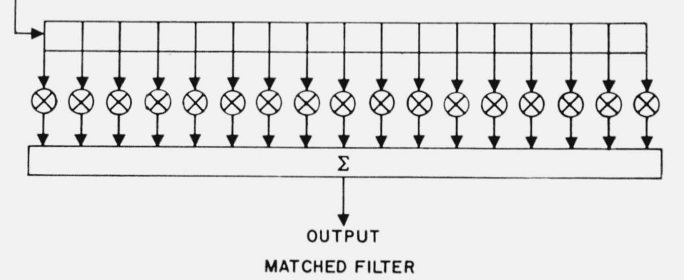

Figure 5. Matched filter.
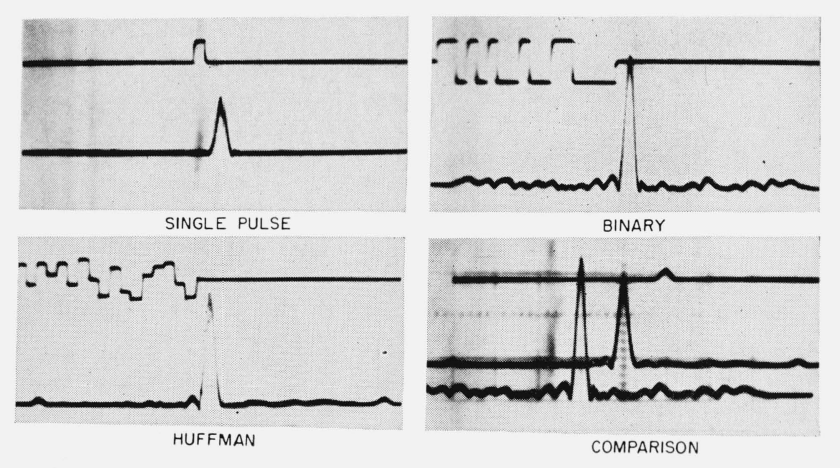

FiguRE 6. Generated waveforms and matched filter outputs. 


\section{Ionospheric Effects on the Signals}

The discussion of signal design, generation, and reception in the previous section has neglected the fact that the signals are to be used for ionospheric sounding. Three effects, due to propagation and ionospheric reflection, are liable to affect the pulse compression capability of the system. They are

(i) Changes in the length of the propagation path during transmission of the signal. Such changes alter the relative phases of the received signal and the receiver local oscillator. That is, in figure 4, $\theta$ becomes a function of time. This nonstationarity of the medium limits the length of signal that may be used.

(ii) Multipath. The superposition of multiple reflections will distort the expected receiver output, due to the squarers in the quadrature detector. This effect limits the unambiguous determination of the relative amplitudes of multipath components.

(iii) Change of the propagation time with frequency. This effect limits the signal bandwidth that may be used.

From a consideration of these points, it is clear that pulse compression will be most effective during times of stable conditions, at frequencies removed from the critical layer frequencies, and will be most useful for the qualitative detection of echoes that would otherwise be obscured by noise. It will work during disturbed conditions, but with reduced efficiency.

\section{Performance}

The signals described above were tested on a vertical sounder at Ottawa, Ontario during September 1963. The sounder was tunable, but was operated at suitable fixed frequencies. The dramatic advantage of the pulse compression signals, i.e., the improvement in signal-to-noise ratio relative to that of a single pulse, is shown in figures 7 and 8 . To make these oscillographs, the system gains were adjusted to equalize the output noise levels. Figure 7 shows A-scan photographs of two transmissions, separated in time by 100 msec. The time bases used were approximately $3 \mathrm{msec}$ long in each case. The echoes that are just barely discernible in the single pulse case are clearly evident in the binary case. Similarly, in figure 8, a Huffman signal and a single pulse are compared, the two photographs being taken 100 msec apart. It is in situations such as these that pulse compression signals will be most useful.

The advantage of impulse-equivalent pulse trains over binary ones, from the point of view of side lobe ambiguity, is demonstrated in figure 9. Here, the response to a Huffman signal is shown on the left and the response to a binary signal transmitted 100 msec later, on the right. The time scale is $200 \mu \mathrm{sec}$ per division. The absence of side lobes on the Huffman signal is evident in the interval between the extraordinary echo (in the middle of the each trace) and the dispersed, high-amplitude ordinary echo (at the right-hand-side of each trace). In the
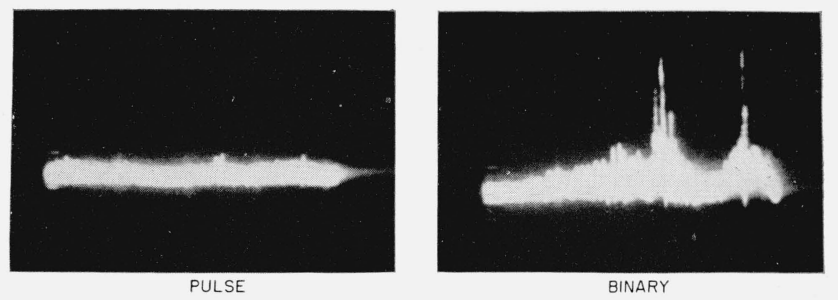

FiguRE 7. Improvement in signal-to-noise ratio.
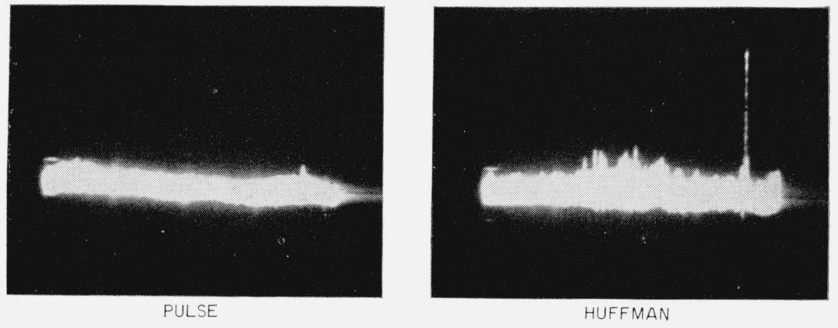

FiguRE 8. Improvement in signal-to-noise ratio.

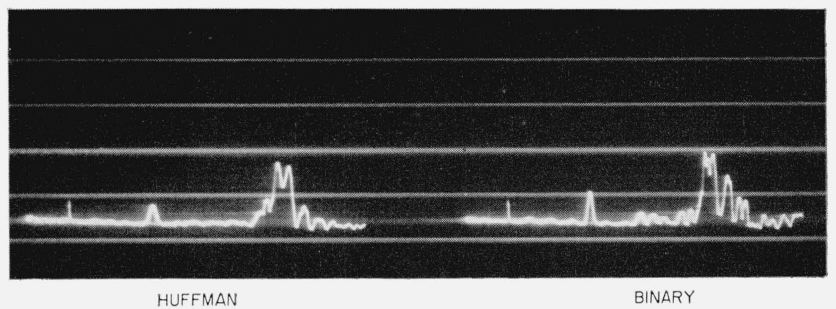

Figure 9. Comparison of Huffman and binary signals.

binary case, it is difficult to distinguish between the extraordinary echo and the side lobes of the ordinary echo.

\section{Conclusions}

Experience gathered with the experimental equipment indicates that a variety of signals should be available for sounding purposes. The signal used at any time would depend on the specific requirement, propagation conditions, and interference environment. To achieve best performance under all conditions, sounders must be able to adapt to meet each new set of conditions.

Single pulses are idealy the best signals for sounding. For those situations in which high signal-tonoise ratios exist single pulses should be used.

Huffman signals may be used to achieve increased detectability with a minimum of side lobe ambiguity. They may also prove useful in providing sounding systems using lower peak powers than the HF sounders now in operation. This would be of particular advantage for mobile sounding installations in satellites or aircraft. 
Binary signals should be used to provide maximum detectability when signal-to-noise ratios are poor. They also are the simplest to instrument, and might find application in digital systems.

In comparing the performance of various signals it is worth noting that if the provision of added energy in the signal, in the form of pulse compression codes with reasonable peak-to-side-lobe ratios, allows the detection of echoes previously obscured by noise, then the problem of sidelobe ambiguity is of secondary importance.

\section{Future Experiments}

The equipment used in this study is being prepared for oblique sounding applications. It will be operated in conjunction with programmable frequency synthesizers to provide stepped frequency sounding from 2 to $24 \mathrm{Mc} / \mathrm{s}$ over a $1000 \mathrm{~km}$ path.

(Paper 68D10-415)

\section{References}

Chapman, J. H., K. Davies, and C. A. Littlewood (1955), Radio observations of the ionosphere at oblique incidence, Can. J. Phys. 33, 713-722.

Coll, D. C., and G. E. Poaps (Jan. 1964), A video signal enhancer for improving the performance of oblique incidence ionospheric sounders, DRTE Technical Report No. 1127.

Cox, J. W., and K. Davies (1955), Oblique incidence pulse transmissions, Wireless Engineer 32, 35-41.

Frank, R. L. (Jan. 1963), Polyphase codes with good nonperiodic correlation properties, IEEE Trans. Inform. Theory IT-9, No. 1, 43-45.

Hatton, W. L. (Sept. 1961), Oblique-sounding and HF radio communication, IRE Trans. Commun. Systems CS-9, No. 3, 275-279.

Huffman, D. A. (Sept. 1962), The generation of impulseequivalent pulse "trains", IRE Trans. Inform. Theory IT-8, No. 5, s10-s 16 .

Jull, G. W., D. J. Doyle, G. W. Irvine, and J. P. Murray (July 1962), Frequency sounding techniques for $\mathrm{HF}$ communications over auroral zone paths, Proc. IRE 50, No. 7, $1676-1682$.

Turin, G. L. (June 1960), An introduction to matched filters, IRE Trans. Inform. Theory ITT-6, No. 3, 311-329.

Warren, E. S. (Nov. 1962), Sweep-frequency radio soundings of the top side of the ionosphere, Can. J. Phys. 40, p. 1692 